\title{
Estimating Risk when Zero Events have been Observed
}

Prof John Quigley, Department of Management Science, University of Strathclyde, Glasgow, UK Dr Matthew Revie, Department of Management Science, University of Strathclyde, Glasgow, UK, Dr Jesse Dawson, Institute of Cardiovascular and Medical Sciences, University of Glasgow, Glasgow, UK

Assessing the risk of complications or adverse events following an intervention presents challenges when they have not yet occurred. Suppose, for instance, a chronic shortage of cardiac telemetry beds has prompted a hospital to implement a new policy that places low risk patients admitted to "rule out myocardial infarction" in regular ward beds (i.e., with no telemetry). After 6 months and the admission of 100 such patients, no cardiac arrests or other untoward events have occurred. This absence of harm (i.e., zero adverse events) indicates a low risk, but clearly we cannot infer a risk of zero on the basis of only 100 patients. But, what can we see about the true underlying risk?

The Rule of Three (Ro3), first proposed by Hanley and Hand [1], provides an estimate for the upper bound of the underlying risk when zero events of interest have occurred through considering the lack of occurrences as moderately rare. The Ro3 estimates the risk as no greater than $\frac{3}{n}$, where $n$ is the number of opportunities or exposures to the risk. Technically, $\frac{3}{n}$ is obtained through assigning the probability 0.95 to measure the likelihood of realising more events than those observed. Note that the Ro3 estimate applies only when one has at least 30 observations. In the above example, the Ro3 would estimate the upper limit for the risk of adverse events associated with the new policy as $\frac{3}{100}$ or $3 \%$.

This estimate does not sound as reassuring as simply stating that no harm has occurred among 100 patients. On the one hand, the real risk could be zero or very close to it. On the other hand, although moderately rare, there is a $5 \%$ chance that a risk as high as $3 \%$ would have resulted in such few occurrences. For practical purposes, one would like not just the "worst case scenario" (i.e. the risk could be as high as $3 \%$ ) but also a sense of the most likely underlying risk, which would be much 
closer to zero. While the Ro3 is still applied today [2], there has been little research on the subject since its development; for a detailed review see [3].

In principle, we could apply this general approach to situations where we have experienced events but simple formulas such as the Ro3 do not exist and would require computer assistance and substantial statistical knowledge to solve. Thus, comparing the risk between interventions that have different number of trials and adverse events remains a challenge. For example, an existing intervention may have a known frequency of 0.001 of a complication while a recently adopted intervention for the same condition has been used 500 times and no complications have been observed. How can we draw a simple comparison between the two interventions to identify which is safer?

While the Ro3 assigns 0.95 to measure the likelihood of realising more events than those observed, Quigley and Revie [4] estimates this probability through minimising the maximum expected error. While numerical methods are required to obtain their estimate, a reasonable approximation can be obtained as follows. Where we have observed $x$ events in $n$ trials, we use two different formulas to estimate the risk; here denoted by $\delta$.

$$
\delta \simeq \begin{cases}\frac{2}{5 n}, & \text { when } x=0 \\ \frac{x}{n}, & \text { when } x \geq 1\end{cases}
$$

It is well known within mathematical statistics that the most accurate estimate of a probability is the Maximum Likelihood Estimator (MLE), i.e. $\frac{x}{n}$ [5]. Simply, the most likely estimate of a probability is the proportion of occurrences observed. When $x$ events occur in $n$ trials, while a range of possible values exist for the true underlying probability, the most likely value is $\frac{x}{n}$. Unfortunately, this intuitively plausible estimate does not apply when $x$ is zero. Returning to the above example, while we may well believe the risk of adverse cardiac events associated with the new policy is quite low, we cannot justify saying that the "most likely estimate is that there is zero risk." In general, saying that the most likely risk is $\frac{0}{n}$ will be inaccurate and uninformative for decision makers. 
The method proposed in (1) provides a non-zero estimate when zero events have been observed, i.e. $\frac{2}{5 n}$ and is $\frac{x}{n}$ for all other situations. As such, (1) provides a simple and consistent framework through which to compare different interventions

Two illustrative examples are used to outline the benefit of using (1) compared with the Ro3.

Example 1: A nationwide audit was carried out on all deaths from sepsis to explore whether any were preventable through more rapid initiation of sepsis management protocols. From this, it was estimated that 1 in every 100 cases was avoidable, i.e. the risk was 0.01 . A single hospital has made substantial changes to management of such patients and has observed no preventable fatalities in a study of 50 patients. The Ro3 suggests that the risk is between 0 and $\frac{3}{50}=0.06$. However, using (1), the best estimate of the risk is 0.008 .

Example 2: A serious incident occurred in a large hospital following incorrect placement of a feeding nasogastric tube. In the past 6 months (when it is believed a similar complication also occurred) it is estimated that 500 such tubes were inserted and as a result, the risk is estimated to be $\frac{2}{500}=0.004$. A change to insertion policy was made which required a radiology consultant to review a chest $x$-ray to confirm tube positioning immediately and prior to use. In the ensuing 2 months, 125 tubes were inserted with no known complications. The hospital is reviewing it's procedures and is keen to assess if the added cost of the radiologist reviewing the $\mathrm{x}$-ray reduces the risk. The estimate using the Ro3 is 0.024 while the estimate using (1) is 0.0032 .

In both examples we use the Ro3 and (1) for different purposes. The Ro3 suggested insufficient information had been collected to conclude that either of the new interventions were improvements. As the Ro3 assesses a 'maximum risk', we would require excessive amounts of data to conclude otherwise. However, (1) provided a more accurate estimate suggesting each new procedure resulted in a reduction of risk.

The Ro3 can be extended to estimate risk for situations where events have been realised; hence facilitating comparisons between risks, such as those in example 2. However, simple accessible formulas for these situations do not exist, unlike with the method proposed in (1), which would support simple, timely comparisons.

To conclude, one key shortcoming of the Ro3 is a loss of simplicity for non-zero data and so is difficult to use when making comparisons across different interventions. A second is, when 
combining probabilities the level of pessimism is compounded beyond that specified at the $5 \%$ level of the Ro3. Thus, using the Ro3 when making comparisons between interventions, decision makers may mix methods resulting in incoherent assessments. We have proposed a simple formula which is an accurate assessment of risk as well as coherently supporting comparisons between risks.

\section{References}

[1] - Hanley, J., and A. Lippman-Hand. "If Nothing Goes Wrong, Is Everything All Right? Interpreting Zero Numerators." Journal of the American Medical Association 249, no. 13 (1983 , JAMA, 249 (13): pp 1743-1745): 1743-45.

[2] - Upile, T, J Mahil, and W Jerjes. "Advances in Consenting: The Rule of 3 and Its Modifications for the Surgeon." Clinical Otolaryngology 37, no. 2 (2012): 165-66.

[3] - Freeman, Raymond. "What to Do When Nothing Has Happened?". Process Safety Progress 30, no. 3 (2011): 204-11.

[4] - Quigley, J., and M. Revie. "Estimating the Probability of Rare Events: Addressing Zero Failure Data." Risk Analysis 31, no. 7 (2011): 1120-32.

[5] - Arnold, S.F. (1990), Mathematical Statistics. Prentice-Hall, 1990. 